\title{
Distribución espacial del riesgo potencial de marchitamiento del aguacate causado por Phytophthora cinnamomi en la subregión de Montes de María, Colombia
}

\author{
Spatial distribution of the potential risk for avocado wilting \\ disease, caused by Phytophthora cinnamomi in the subregion \\ of Montes de Maria, Colombia
}
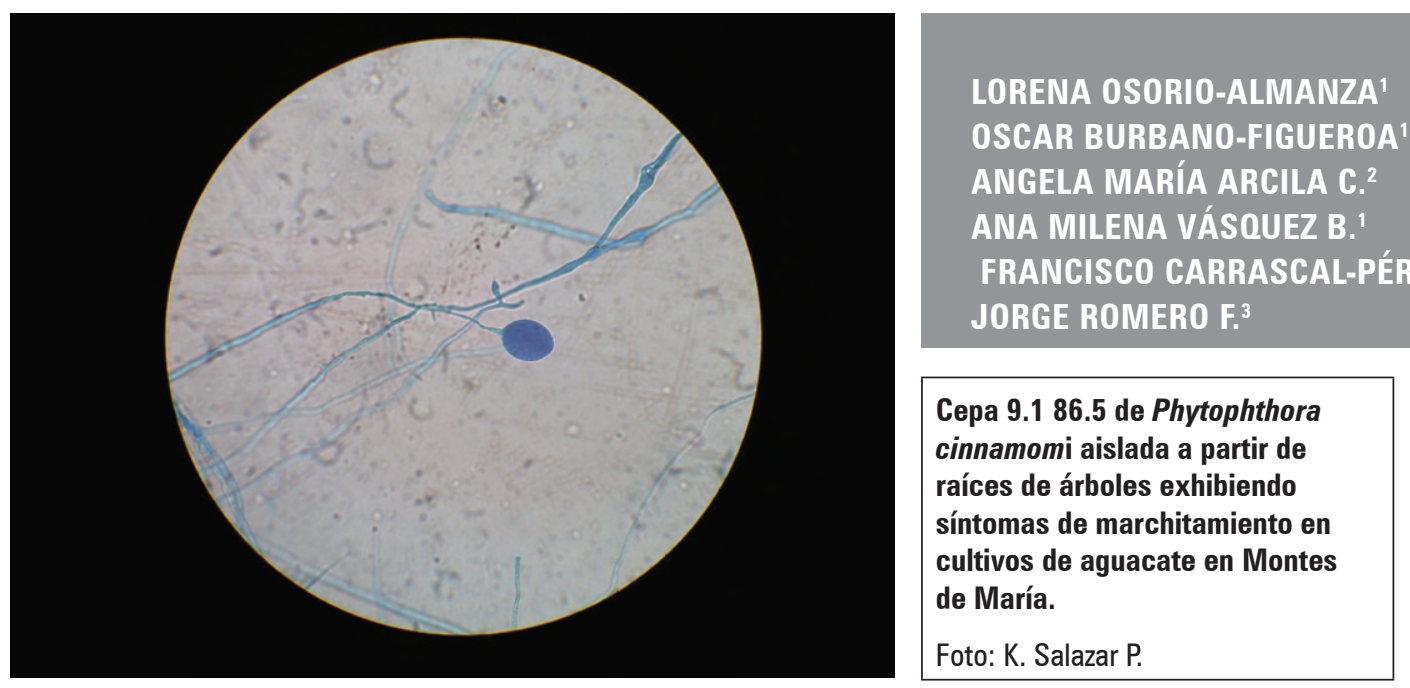

\section{RESUMEN}

El marchitamiento del aguacate, causado por el oomycete Phytophthora cinnamomi Rands, es la enfermedad más limitante del cultivo a nivel mundial. Montes de María (MM) es la principal región productora de aguacate del Caribe Colombiano. El área de producción de aguacate en esta región, se encuentra en declive en contraposición a la tendencia mundial y nacional. Esta disminución en $\mathrm{MM}$ ha sido asociada a problemas sanitarios, específicamente al marchitamiento del aguacate ocasionado por P. cinnamomi. El clima es uno de los factores más importantes que determinan la distribución espacial de una especie y en el caso de los patógenos determina el nicho que facilita la colonización del hospedero. En este contexto, se tiene como propósito estimar la distribución espacial del riesgo de marchitamiento del aguacate en la subregión Montes de María. Esta estimación se realizó a partir de los datos de presencia del síntoma de marchitamiento de yemas, característico de la pudrición radicular por P. cinnamomi. Este síntoma exhibió una alta correlación con la incidencia y

Centro de Investigación Turipaná, The Plant Interactions Laboratory, Corporación Colombiana de Investigación Agropecuaria (Corpoica), Cereté (Colombia). ORCID Osorio-Almanza, L.: 0000-0002-7520-4716; ORCID Burbano-Figueroa, O.: 0000-0002-6604-7333; ORCID Vásquez-B., A.M.: 0000-0001-6435-8464

2 Centro de Investigación Caribia, Laboratorio de Entomología, Corporación Colombiana de Investigación Agropecuaria (Corpoica), Sevilla (Colombia). ORCID Arcila, A.M.: 0000-0001-7353-5207; ORCID Carrascal-Pérez, F.: 0000-00017353-5207

3 Centro de Investigación Turipaná, Laboratorio de Fisiología Vegetal, Corporación Colombiana de Investigación Agropecuaria (Corpoica), Cereté (Colombia). ORCID Romero F., J.: 0000-0002-7249-6549

4 Autor para correspondencia. oburbano@corpoica.org.co 
la severidad de la enfermedad evaluada por fincas. El modelo Maxent usado para estimar la distribución espacial de riesgo muestra una alta validación estadística (test $A U C \geq 0,9$ ). Este modelo ha permitido predecir las zonas donde el riesgo a pudrición radicular es más alto. Las características de las áreas con el mayor riesgo de pudrición radicular son aquellas localizadas a mayor altitud, con las temperaturas más bajas en el trimestre más frío (BIO11 $\left.\sim 22^{\circ} \mathrm{C}\right)$ y en el más caluroso (BIO10 $\sim 24^{\circ} \mathrm{C}$ ) y con la precipitación más alta durante la estación seca (BIO17 - BIO14).

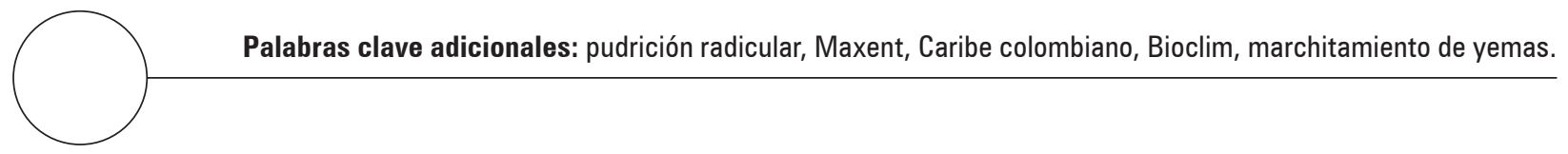

\section{ABSTRACT}

Avocado wilting or root rot, caused by the oomycete Phytophthora cinnamomi Rands, is the most limiting disease of avocado crops around the world. Montes de Maria (MM) is the main producer region of avocado in the Colombian Caribbean. Avocado producing areas in MM are decreasing in an inverse trend compared with the increasing hectarage world and nation-wide. Decreasing hectarage in MM is associated with phytosanitary problems, specifically the avocado wilting caused by P. cinnamomi. Climate is one of the main abiotic factors determining spatial distribution of species. For plant-pathogens, environmental conditions determine host-colonization. Under this scenario, the aim of this study was to estimate the spatial distribution of the avocado root-rot risk in the subregion of Montes de Maria, Colombia. This estimation was based on data for the presence of shoot dieback, a characteristic, easily identifiable symptom of root rot caused by $P$. cinnamomi. This symptom exhibits a high correlation with disease incidence and severity. A Maxent model was used to estimate the spatial distribution of the risk, showing a high statistical validation (test AUC>0.9). This model identified areas with a high risk for occurrence of branch dieback and, consequently, root rot. Areas with a higher risk of root rot were found at the higher altitudes, with lower temperatures in the coldest trimester (BIO11 $\sim 22^{\circ} \mathrm{C}$ ), and in the warmest one (BIO10 $24^{\circ} \mathrm{C}$ ), with higher rainfall during the dry season (BIO17 - BIO14).

Additional key words: root rot, Maxent, Colombian Caribbean, Bioclim, shoot dieback.

Fecha de recepción: 16-03-2017 Aprobado para publicación: 30-09-2017

(

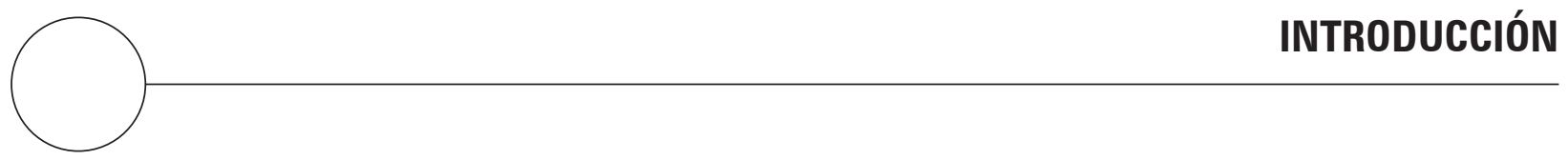

La subregión Montes de María (MM) se encuentra ubicada en el centro de los departamentos de Bolívar y Sucre, en la región Caribe Colombiana, e integrada por 15 municipios: El Carmen de Bolívar, San Jacinto, San Juan Nepomuceno, El Guamo, María la Baja, Zambrano, Córdoba (Bolívar), Ovejas, Los Palmitos, Morroa, Colosó, Chalán, Toluviejo, San Onofre y San Antonio de Palmito (Sucre). Los MM tienen una extensión de $6.297 \mathrm{~km}^{2}$ aproximadamente con un clima de sabana tropical con invierno seco (Aw) según la clasificación climática de Köppen, temperatura promedio anual de $27^{\circ} \mathrm{C}$ con periodos alternados de sequía (verano) y humedad (invierno) durante todo el año (Kottek et al., 2006). MM se caracteriza por presentar una gran diversidad geomorfológica desde montañas con altas pendientes hasta zonas de planicie alcanzando elevaciones superiores a los $650 \mathrm{msnm}$. La subregión presenta múltiples microcuencas que están inmersas en la gran cuenca de los ríos Magdalena y San Jorge. Esta característica le confiere al territorio aptitud para la producción agrícola (Téllez, 2015). Los principales cultivos de la subregión son yuca, name, tabaco, palma de aceite, cacao y aguacate. Estos cultivos representan una fuente significativa de ingresos a la población agricultora y comercializadora.

El aguacate es uno de los cultivos de mayor importancia económica en la subregión de MM. En esta 
subregión predominan variedades silvestres (landraces) de la raza antillana, principalmente árboles de gran altura que forman parte de la vegetación de bosques de la región (Vega, 2012). En realidad, la población de aguacate de MM no es manejada por los agricultores como un cultivo, sino como un bosque donde los frutos son recogidos durante la temporada de cosecha (Burbano-Figueroa, comunicación personal). Las poblaciones de aguacate de la raza antillana, exhiben alturas superiores a $10 \mathrm{~m}$ y su copa puede llegar a alcanzar los $25 \mathrm{~m}$ de diámetro. Los frutos de las variedades antillanas son de gran tamaño, alcanzando los $2.500 \mathrm{~g}$ de peso y son de formas ovaladas, redondas o piriformes. La raza antillana está adaptada a climas tropicales secos con temperaturas de 18 a $26^{\circ} \mathrm{C}$ y alturas inferiores a los 1.000 msnm y probablemente su centro de origen son las tierras bajas de Guatemala (Chen et al., 2009). En Colombia, las siguientes variedades pertenecen a la raza antillana: Butler, Fuchs, Fucsia, Hulumanu, Lorena, Peterson, Pinelli, Pollock, Ruehle, Russe11, Simmonds, Trapp, Villacampa, Waldin, Común o Criollo, Venezolano y Curumaní (DANE, 2015; Vega, 2012; Tamayo et al., 2008).

Las áreas de cultivo en MM se encuentran en declive en contraposición a la tendencia mundial y nacional de aumento de áreas sembradas. En Colombia, el área del cultivo ha aumentado de 30.006 ha en 2010 a 45.070 ha en el 2013, incrementando incluso el número de toneladas de exportación pasando de 56 t en 2010 a 3.373 t para el 2015 (Pavas, 2015). El declive en las áreas de cultivo en los MM ha sido asociado a problemas sanitarios, específicamente el marchitamiento del aguacate ocasionado por $P$. cinnamomi. Para el año 2004, la región de MM alcanzó el máximo histórico de producción con 70.000 t de aguacate. En el año 2010 como resultado de los múltiples problemas sanitarios y de manejo del cultivo, la producción se redujo a 38.000 t. Esta misma tendencia de disminución de la producción ha sido observada en el área de cultivo. El área sembrada de aguacate en los MM disminuyó desde 3.000 ha en el año 2007 a 1.723 en 2013 (Vega, 2012).

El marchitamiento o pudrición radicular del aguacate es causada por el oomycete P. cinnamomi Rands. Es la enfermedad más limitante del aguacate a nivel mundial (Zentmyer, 1981). Los árboles infectados exhiben clorosis, producción de hojas pequeñas, defoliación y en los estados más avanzados de la enfermedad; marchitamiento de yemas y muerte uno o dos años, después iniciada la infección. Estos síntomas son más notorios y capaces de detener el crecimiento del árbol bajo condiciones de estrés, especialmente el causado por la escasez de agua (Coffey, 1987). Suelos con pobres drenajes, alto contenido de arcillas, o áreas donde el agua se acumula después de la irrigación o la lluvia han sido históricamente asociados con altos niveles de severidad de pudrición radicular causada por $P$. cinnamomi (Burns et al., 1960). P. cinnamomi es un patógeno del suelo capaz de parasitar múltiples hospederos en ecosistemas agrícolas, hortícolas y forestales y sobrevivir en el suelo como saprófito. Estas características hacen que una vez que el patógeno esté establecido en el suelo, su erradicación sea muy compleja (Pérez-Jiménez, 2008; Zentmyer y Mircetich, 1967). $P$. cinnamomi puede esparcirse a través del suelo en los equipos del cultivo, en el agua de escorrentía e infiltración, o por la siembra de material infectado (Aubrey, 1980; Erwin y Ribeiro, 1996; Pegg et al., 2002). La medida de control más efectiva es evitar la contaminación con P. cinnamomi sembrando plantas de invernadero libres del patógeno en suelos limpios (Erwin y Ribeiro, 1996; Pegg et al., 2002). En áreas donde la pudrición radicular está presente, el uso de patrones resistentes es el mecanismo más efectivo para controlar la enfermedad (Zentmyer, 1984). A excepción de los fosfonatos (fosetil-Al o ácido fosforoso), los fungicidas no son efectivos en el control de la enfermedad. Los fosfonatos usados en drench en el suelo, aspersiones foliares, o inyecciones en el tronco, son capaces de controlar la enfermedad con un costo razonable (Coffey, 1987; Darvas et al., 1983; 1984; Pegg et al., 2002). Una estrategia integrada de control efectiva y sostenible de esta enfermedad requiere material vegetal de siembra en buenas condiciones sanitarias, suelos bien drenados, y manejo del cultivo adecuado, especialmente irrigación, uso de patrones resistentes y control químico con fungicidas apropiados (Erwin y Ribeiro, 1996; Pegg et al., 2002).

La mayoría de patrones disponibles pertenecen a la raza mexicana y en consecuencia, no están adaptados para las temperaturas del trópico bajo. Estos patrones exhiben tolerancia a la enfermedad y a otros factores del suelo. En Colombia, no existen patrones antillanos de resistencia conocida a la pudrición radicular. Es frecuente que plántulas de landraces de aguacate de la raza Antillana provenientes de Montes de María, sean usados como patrones para variedades de aguacate adaptadas a climas templados (raza Guatemalteca) especialmente de la variedad Lorena y Choquette (Tamayo et al., 2008; Vega, 2012). Un centenar de 
estos individuos identificados en áreas donde la severidad de la pudrición radicular es alta han sido seleccionados para futuras evaluaciones de resistencia (Romero-Ferrer y Burbano-Figueroa, com. pers.).

Los modelos de estimación de nicho ecológico están fundamentados en el concepto de la ecología clásica de "nicho" y en el potencial de desarrollar modelos siguiendo este concepto con el propósito de establecer la distribución de una especie (Jiménez-Valverde et al., 2008; 2011). Un tipo de estos modelos, son los modelos correlativos de relaciones entre la ocurrencia de una especie en una localidad específica y las características ambientales de esa localidad. Los modelos correlativos son usados ampliamente para evaluar el riesgo de establecimiento de especies de plagas y enfermedades de cultivos (Bogosian et al., 2012; Kumar et al., 2014). Uno de los modelos correlativos más frecuentemente usados es MaxEnt. Este algoritmo que permite "estimar la probabilidad de distribución de Máxima Entropía, indicando la concentración del modelo alrededor de las muestras de presencia y si dicha concentración es mayor que en un pixel aleatorio" (Phillip et al., 2005). Además del modelo de Máxima Entropía, también se emplean otros algoritmos como Mahalanobis y regresión logística. Estos modelos son capaces de predecir más del $50 \%$ de la presencia de una enfermedad o sus síntomas en las localidades seleccionadas (Galdino et al., 2016; La Manna et al., 2012; Guevara et al., 2016).

En el actual escenario de MM, comprender los factores asociados al riesgo de establecer cultivos de aguacate en áreas proclives al marchitamiento por pudrición radicular es urgente para desarrollar estrategias adecuadas de manejo que faciliten el establecimiento de nuevos cultivos de aguacate y el control efectivo de la enfermedad en los cultivos ya establecidos. El clima es uno de los factores abióticos más importantes que determinan la distribución espacial de una especie y en el caso de los patógenos, regula la colonización de un hospedero. Los modelos de nicho ecológico correlativos basados en las relaciones entre variables ambientales y ocurrencia de especies son usados para predecir posibles áreas de introducción de patógenos, establecimiento y dispersión de estos. En este escenario, este trabajo tiene como propósito estimar la distribución espacial del riesgo de marchitamiento del aguacate en la subregión de Montes de María, Colombia, y determinar las áreas de MM que exhiben el mayor riesgo para el desarrollo de la enfermedad.

\section{MATERIALES Y MÉTODOS}

\section{Área de estudio}

La zona objeto de estudio se encuentra enmarcada en la subregión Montes de María, y es definida con mayor exactitud como la zona con altitudes que superan los 148 msnm según el modelo digital de elevación y en la cual se encuentra establecida la mayor parte de las áreas del cultivo de aguacate. La región presenta suelos inceptisoles. Estos se caracterizan por presentar un elevado contenido de arcilla, siendo las texturas dominantes franco arcillosa, franco arcillo arenosa y arcillosa (Chinchilla et al., 2011).

\section{Datos de severidad y ocurrencia de pudrición radicular}

Los datos de severidad y ocurrencia de la enfermedad fueron estimados a partir de trabajo de campo desarrollado para evaluar el impacto de plagas y enfermedades en el cultivo de aguacate en la región Caribe. En este trabajo de campo, se evaluaron 460 árboles distribuidos en 46 fincas, e identificaron 228 árboles de aguacate con diferentes niveles de severidad de la enfermedad y en 39 de ellos fue identificada la presencia de P. cinnamomi. Entre los árboles exhibiendo síntomas de la enfermedad, 82 árboles mostraron marchitamiento de yemas. Las fincas fueron visitadas entre 2014 al 2015. Las fincas seleccionadas corresponden a fincas con reportes previos de la presencia de pudrición radicular.

Incidencia (INC) y severidad de la pudrición radicular fue estimada a partir de evaluaciones de campo en trayectos de $100 \mathrm{~m}$ (aproximadamente 10 árboles) para cada una de las 46 fincas usando la escala descrita a continuación: (1) Amarillamiento de hojas (AM), u hojas cloróticas; (2) Márgenes necróticos en las hojas (HN) y (3) Pequeñas ramas exhibiendo marchitamiento en la parte superior del árbol o defoliación intensa en las ramas superiores (MY: marchitamiento de yemas). El índice de severidad (SEV) para cada finca fue estimado como la suma de los puntajes asignados a cada uno de los árboles en concordancia con el síntoma de mayor deterioro sanitario exhibido desde 1 hasta 3 , donde 3 representa la peor condición sanitaria (MY) y 1 la menor (AM).

La correlación entre la incidencia, severidad y la frecuencia de cada uno de los niveles de desarrollo de 

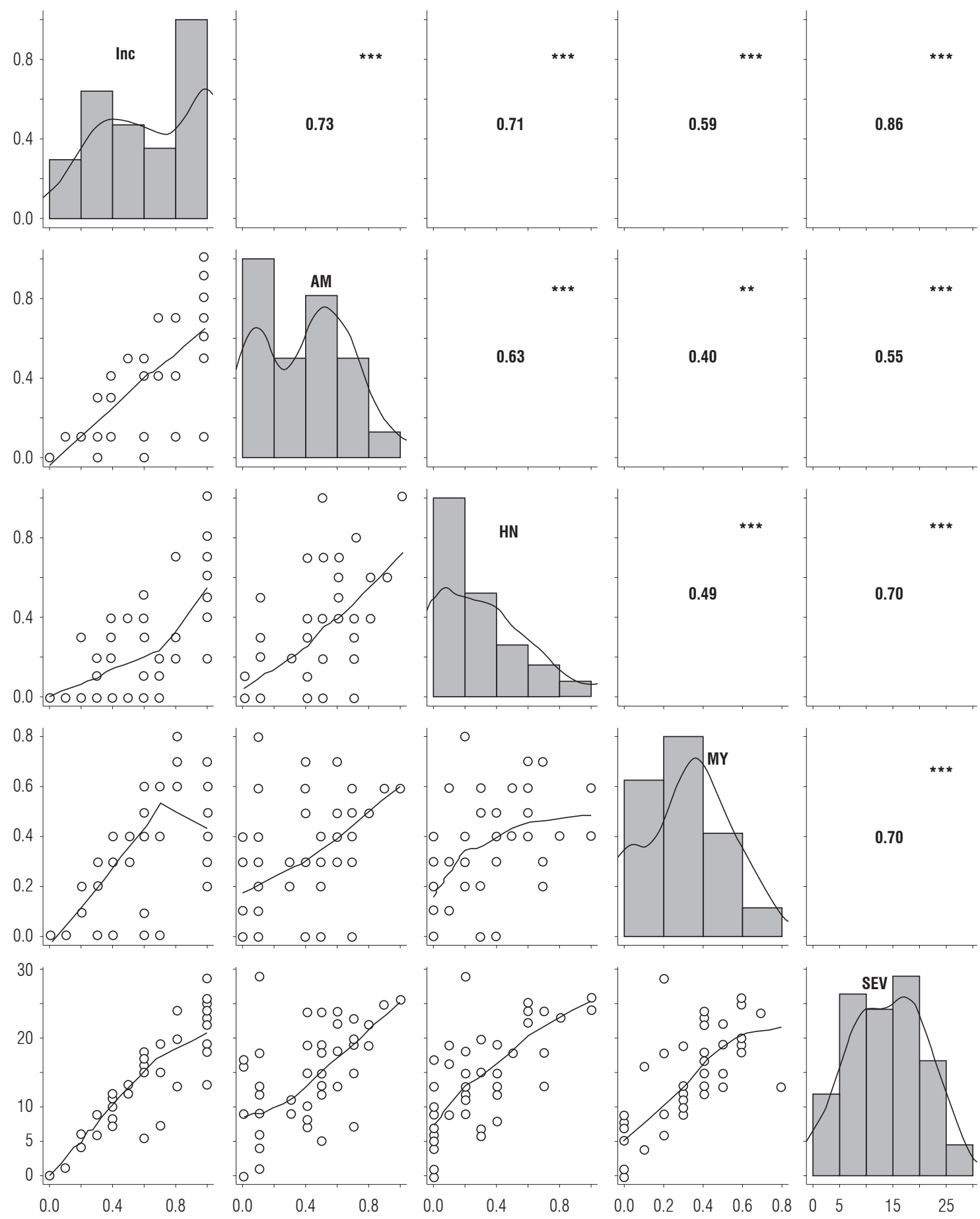

Figura 1. Correlación de incidencia, frecuencia de síntomas y severidad de pudrición radicular en aguacate en fincas de la subregión Montes de María, Colombia. Inc. (Incidencia), AM (Amarillamiento de hojas), HN (Márgenes necróticos en las hojas) y MY (Marchitamiento de yemas) están expresados como frecuencias relativas, y SEV (Severidad) en valores adimensionales (descripción en materiales y métodos). Las correlaciones significativas están marcadas con asteriscos que indican los valores de probabilidad: ${ }^{* *} \leq 0,001 ; *{ }^{*} \leq 0,01 ; * 0,05$. 
la enfermedad fue evaluada por el método de correlación de Pearson usando el paquete R (versión 3.2.2). El propósito de determinar tales correlaciones fue establecer la existencia de una relación lineal entre la incidencia y la severidad de la enfermedad, y en caso positivo determinar si el marchitamiento de yemas exhibe una correlación significativa con estos parámetros. En consideración a la correlación significativa mostrada entre MY e INC y SEV (Fig. 1), la ocurrencia de la enfermedad fue restringida exclusivamente a las evaluaciones positivas por finca del MY. Para evaluar la importancia de las variables ambientales, estas fueron sometidas a la prueba de Jackknife bajo dos evaluaciones, una de Regularized training gain y el Test AUC. Este Test o cálculo de área bajo la curva permite estimar la capacidad de predicción del modelo.

El marchitamiento de yemas es el síntoma más característico de la pudrición radicular causada por
P. cinnamomi, y es evidencia de un estado avanzado de deterioro de la sanidad de los árboles de aguacate (Coffey, 1987). En este sentido, considerar únicamente el MY como estimación de la ocurrencia de la enfermedad evita la sobreestimación de la enfermedad, al no incluir los síntomas más leves asociados al estrés hídrico y disminuir la autocorrelación asociado a la longitudinal espacial de los datos de los árboles provenientes de una misma finca.

\section{Variables ambientales}

Para el modelamiento de nichos es necesario hacer uso de las variables ambientales que inciden sobre la zona objeto de estudio, por lo que se tuvo en cuenta información bioclimática (Tab. 1). Las capas climáticas fueron obtenidas de WorldClim (Hijmans et al., 2016) que es un set de capas climáticas a nivel global con

Tabla 1. Variables ambientales consideradas en el modelo de nicho para $P$ cinnamomi Rand y contribución relativa de las variables ambientales en el model Maxent de riesgo potencial del marchitamiento del aguacate en la subregión Montes de María.

\begin{tabular}{|c|c|c|c|c|c|c|}
\hline Variables & $\begin{array}{l}\text { Porcentaje de } \\
\text { contribución }\end{array}$ & $\begin{array}{l}\text { Importancia de } \\
\text { permutación }\end{array}$ & Min. & Máx. & Promedio & $\begin{array}{c}\text { Desviación } \\
\text { estándar }\end{array}$ \\
\hline Altitud & 29,5 & 0 & 1,2 & 774,5 & 351,5 & 223,9 \\
\hline Precipitación en el período más seco (BI0 14) & 20 & 34,1 & 2,3 & 35,8 & 19,0 & 9,6 \\
\hline $\begin{array}{l}\text { Temperatura promedio en el trimestre más caluroso } \\
\text { (BIO 10) }\end{array}$ & 8,3 & 4,7 & 23,6 & 29,6 & 26,6 & 1,7 \\
\hline Temperatura promedio en el trimestre más frío (BI0 11) & 7,1 & 0 & 22,5 & 28,1 & 25,3 & 1,6 \\
\hline Temperatura media en el trimestre más lluvioso (BI0 8) & 6,3 & 0 & 229,3 & 285,7 & 257,5 & 16,3 \\
\hline Precipitación en el trimestre más seco (BIO 17) & 4,6 & 0 & 7,0 & 163,0 & 85,0 & 45,1 \\
\hline Precipitación en el trimestre más frío (BIO 19) & 4,2 & 2,5 & 7,0 & 607,0 & 307,0 & 173,7 \\
\hline Precipitación en el trimestre más caluroso (BI0 18) & 3,8 & 25,3 & 84,0 & 456,0 & 270,0 & 107,7 \\
\hline Rango anual de temperatura (BI05-BI06) (BI0 7) & 3,6 & 25,5 & 109,6 & 162,4 & 136,0 & 15,2 \\
\hline Precipitación anual (BI0 12) & 3,4 & 0 & 842,0 & 1898,0 & 1370,0 & 305,7 \\
\hline Temperatura promedio anual (BI0 1) & 2,2 & 0 & 231,2 & 288,8 & 260,0 & 16,6 \\
\hline $\begin{array}{l}\text { Rango medio diurno (temp max - temp min; promedio } \\
\text { mensual) (BIO2) }\end{array}$ & - & - & 85,9 & 123,1 & 104,5 & 10,7 \\
\hline Isotermalidad (BI01/BI07)* $100(\mathrm{BIO3)}$ & - & - & 74,5 & 80,5 & 77,5 & 1,7 \\
\hline $\begin{array}{l}\text { Estacionalidad en temperatura (coeficiente de variación) } \\
\text { (BIO4) }\end{array}$ & - & - & 304,4 & 639,8 & 472,1 & 97,1 \\
\hline Temperatura máxima del período más caliente (BI05) & - & - & 293,7 & 369,3 & 331,5 & 21,8 \\
\hline Temperatura mínima del período más frío (BI06) & - & - & 164,2 & 221,8 & 193,0 & 16,6 \\
\hline Temperatura promedio en el trimestre más seco (BI09) & - & - & 229,9 & 291,1 & 260,5 & 17,7 \\
\hline Precipitación en el período más lluvioso (BI013) & - & - & 106,6 & 315,4 & 211,0 & 60,4 \\
\hline $\begin{array}{l}\text { Estacionalidad de la precipitación (coeficiente de } \\
\text { variación) (BI015) }\end{array}$ & - & - & 72,9 & 140,1 & 106,5 & 19,4 \\
\hline Precipitación en el trimestre más lluvioso (BI016) & - & - & 326,7 & 750,3 & 538,5 & 122,6 \\
\hline
\end{tabular}


una resolución espacial de $1 \mathrm{~km}^{2}$ aproximadamente, cuyos datos pueden ser utilizados para el modelado y mapeado en Sistemas de Información Geográfica (SIG). Estas capas de información corresponden a las décadas de 1970 - 2000 (Hijmans et al., 2005).

\section{Construcción del modelo basado en marchitamiento de yemas}

Para el procesamiento de los datos e información obtenida a partir de fuentes primarias (trabajo de campo) y secundarias (BIOCLIM) se empleó el Modelo de Distribución de Especies de Máxima Entropía (MAXENT), el cual en comparación con otros modelos tiene las ventajas las siguientes: 1) sólo requiere registros de presencia, 2) emplea e interactúa con variables continuas y categóricas, 3) la información que se genera es continua permitiendo distinguir predicciones en diversas áreas, 4) el modelo es aplicable a registros de presencia/ausencia, 5) es un modelo generativo, lo cual resulta ser conveniente cuando no se dispone de gran cantidad de datos y 6) puede ser aplicado en diferentes escalas y campos de estudio (Castillo, 2008).

Las capas previamente mencionadas y las coordenadas geográficas obtenidas en campo se procesaron (recorte de capas ráster, conversión de formatos y georeferenciación de las capas) en el programa Quantium GIS, con el objetivo de generar posteriormente un modelo de distribución en el programa de modelamiento MAXENT y en el lenguaje de programación estadística R. Esto permitió estimar la presencia de Phytophthora y el riesgo de marchitamiento por pudrición radicular en la zona de cultivo en la subregión de Montes de María.

Para la construcción del modelo de riesgo, inicialmente se realizó el modelamiento de distribución de árboles de aguacate en la subregión Montes de María, para lo cual se emplearon las variables ambientales mencionadas y las respectivas coordenadas geográficas. Posteriormente, se realizó el modelamiento de probabilidad de ocurrencia de Phytophthora, en el cual se incluyó la capa de distribución de árboles de aguacate generado, así como las capas de variables ambientales descritas y los puntos geográficos donde hay riesgo de la enfermedad. Finalmente, se construyó el modelamiento de marchitamiento de yemas, donde se tuvieron en cuenta las capas mencionadas y las coordenadas geográficas donde se observó sintomatología de la enfermedad y/o marchitamiento de yemas.

\section{RESULTADOS Y DISCUSIÓN}

\section{Incidencia, niveles de la enfermedad y severidad}

Correlaciones significativas fueron encontradas entre la incidencia, severidad y niveles específicos de la enfermedad $(P \leq 0,01)$. El marchitamiento de yemas, un síntoma fácilmente discernible en el campo y claramente asociado con la pudrición radicular causado por P. cinnamomi (Vega, 2012), exhibió una correlación significativa con las variables INC y SEV.

\section{Estimación del modelo en Maxent para marchitamiento}

De acuerdo con el modelo estimado, los cultivos de aguacate en MM se distribuyen principalmente de en altitudes superiores a los $233 \mathrm{msnm}$ (Fig. 5), presentando su mayor concentración en las áreas de mayor altitud, aquellas que superan los $335 \mathrm{msnm}$. Las probabilidades de presencia de individuos de aguacate en las zonas de menor altitud son escasas $(\leq 0,5)$. La pudrición radicular en la región se manifiesta en un área aproximada de $97,63 \mathrm{~km}^{2}$ (Fig. 2, Panel A).

El modelo de estimación de la distribución potencial de la enfermedad de pudrición radicular se desarrolló exclusivamente a partir de los datos de marchitamiento de yemas. Este síntoma exhibe correlación directa con INC y SEV, y es fácilmente cuantificable. El modelo realizado a partir de los datos de la sintomatología muestra que la presencia de marchitamiento es más frecuente en el área central de Montes de María. Esta zona está comprendida por las veredas y caseríos de El Limón, Joján, Jojancito, La Sierra, El Cielo, E1 Orejero, Venado, Berruga, Centro Alegre, Berruguita, El Aguacate, Santa Lucía, El Raizal, La Pita, Pozo Oscuro, La Florida y Carocolicito (Fig. 2, Panel B).

Los valores AUC (area under the curve) de los modelos establecidos fueron calculados para los datos de entrenamiento. Si el test AUC es superior a 0,90 esto indica que hay una alta capacidad de Maxent para predecir el área potencial de las poblaciones de aguacate y del riesgo de esas poblaciones de ser afectada por pudrición radicular (Fig. 3). Los valores AUC entre 0,5 y 0,7 muestran una capacidad de predicción del modelo muy débil; 0,7 a 0,8 es considerado un modelo de desempeño aceptable; 0,8 a 0,9 es un buen desempeño y finalmente, un valor AUC superior a 0,9 muestra que el modelo es altamente preciso, o de excelente desempeño (Manuel et al., 2002). 


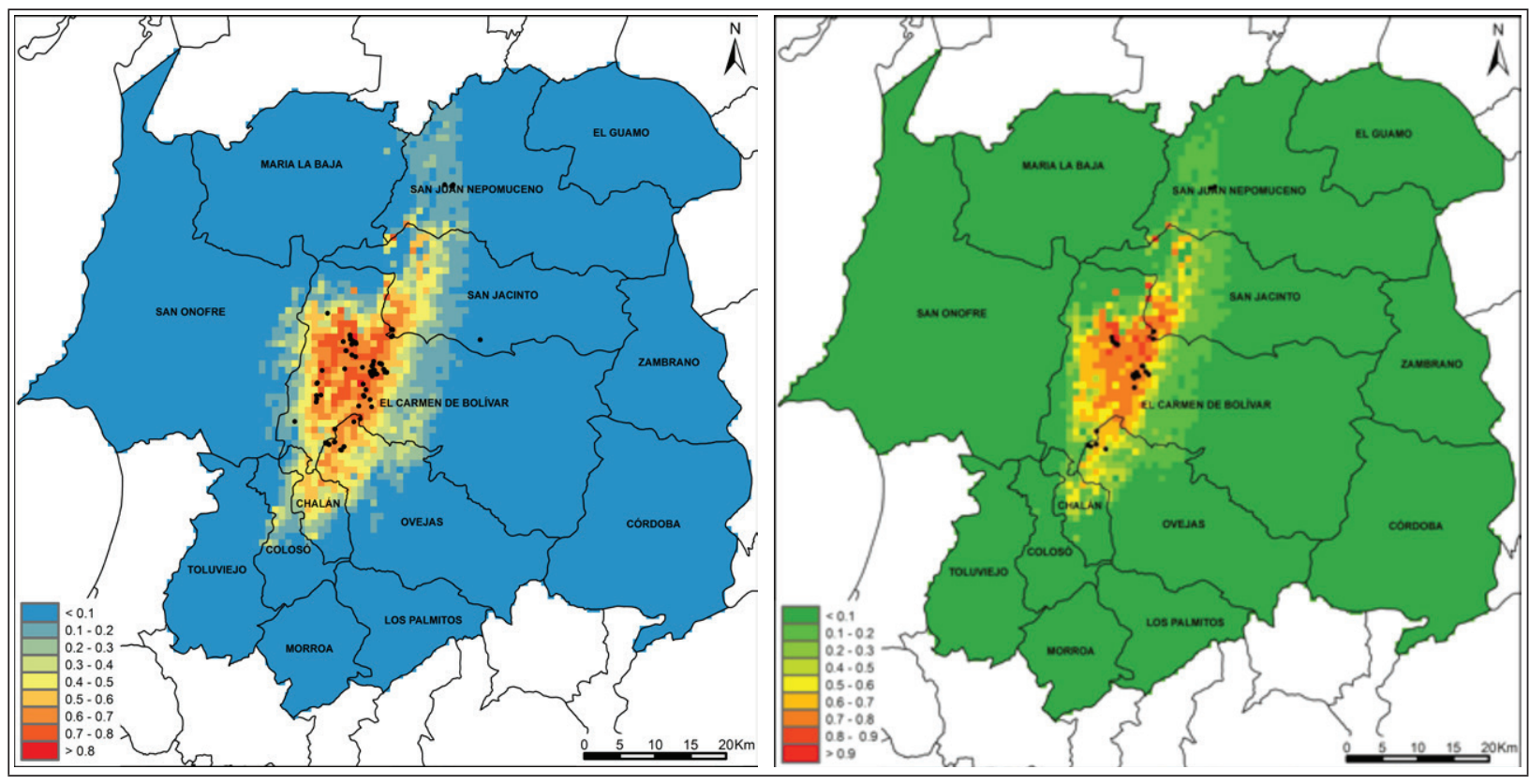

Figura 2. Distribución espacial del riesgo de marchitamiento del aguacate causado por Phytophthora cinnamomi en la subregión de Montes de María, Colombia. Panel A: mapas de distribución potencial de las áreas donde se encuentran árboles de aguacate; Panel B: riesgo de desarrollo de la pudrición radicular del aguacate. La probabilidad de ocurrencia de la enfermedad está determinada por la escala gráfica de acuerdo con la degrades de colores.
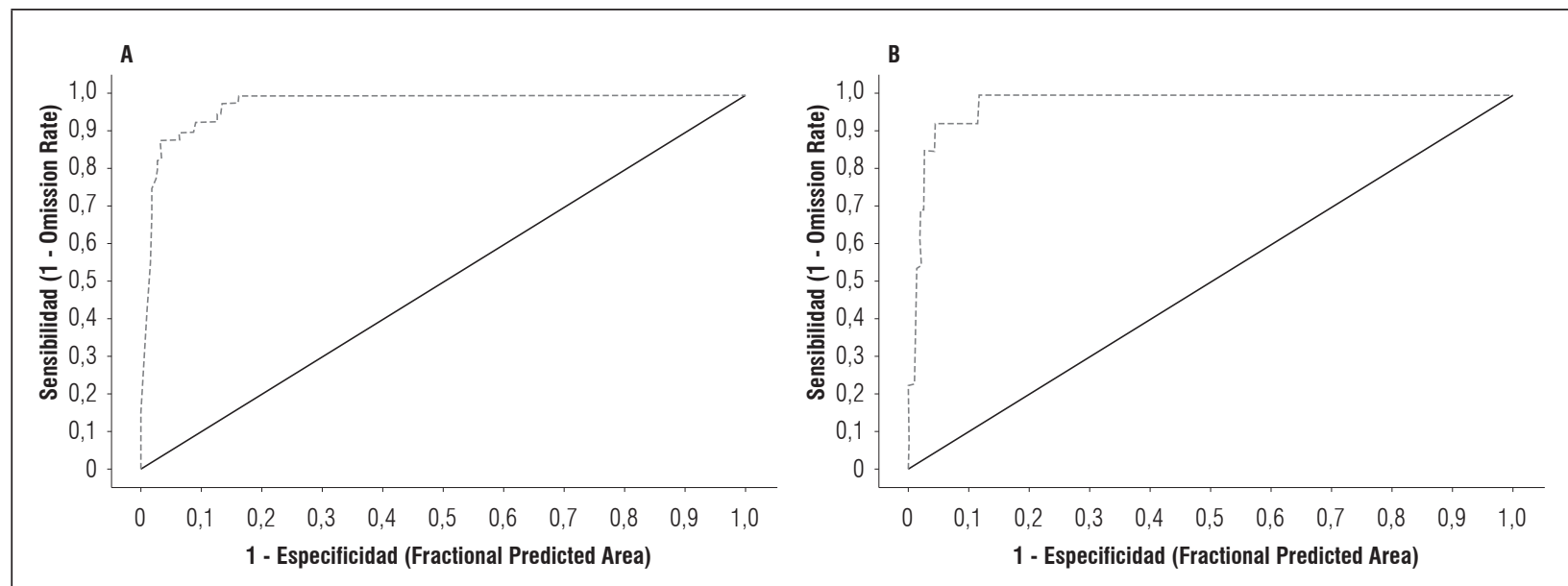

Figura 3. Evaluación mediante área bajo la curva (AUC) para A. Distribución de árboles de aguacate en la subregión Montes de María (AUC = 0,974) y B. Riesgo de marchitamiento del aguacate en la subregión Montes de María $(A U C=0,977)$. Training data (línea punteada), random prediction (línea sólida, AUC $=0,5$ ).

En cuanto a la importancia de las variables ambientales, esta fue evaluada sobre la base de la prueba Jackknife donde los valores indican la contribución de cada una de estas (Fig. 4). Para lo anterior se realizaron dos pruebas, una de Regularized training gain (Panel A) y el Test AUC (Panel B). Con respecto a la importancia de las variables ambientales, la prueba
Regularized training gain, mostró mayor contribución de BIO10, BIO11, BIO8, BIO17 y BIO14, respectivamente; mientras que para el Test AUC, las variables de mayor peso fueron $\mathrm{BIO}$, altitud, BIO10, BIO17, BIO14, BIO11, con porcentajes de contribución dentro del modelo desde el $29,5 \%$ hasta el 4,6\% (Tab. 1). 
A

Temperatura promedio en el trimestre más caluroso (BI0 10)

Temperatura promedio en el trimestre más frío (BI0 11)

Temperatura media en el trimestre más lluvioso (BI0 8)

Altitud
Precipitación en el período más seco (BI0 14)
Precipitación en el trimestre más seco (BI0 17)
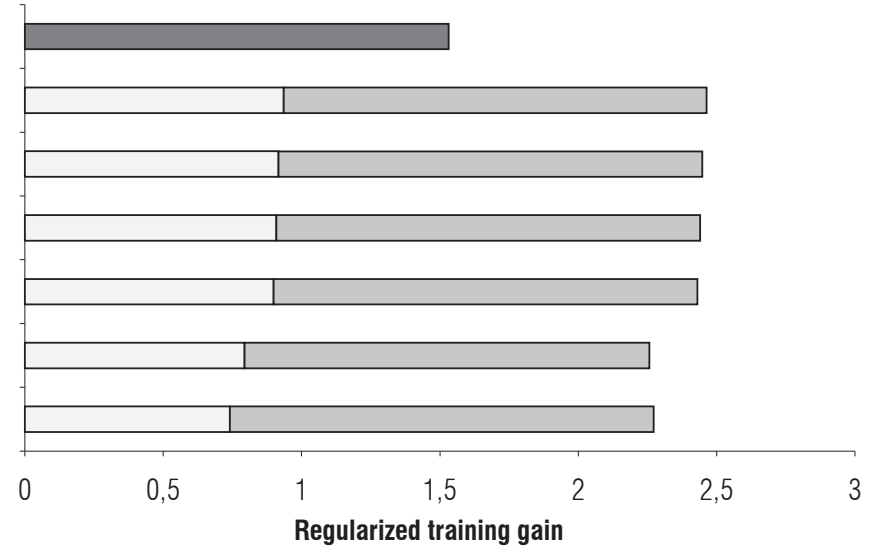

B

Temperatura promedio en el trimestre más caluroso (BI0 10)

Temperatura promedio en el trimestre más frío (BI0 11)

Temperatura media en el trimestre más Iluvioso (BI0 8)

$$
\begin{aligned}
& \text { Altitud } \\
& \text { Precipitación en el período más seco (BI0 14) } \\
& \text { Precipitación en el trimestre más seco (BI0 17) }
\end{aligned}
$$

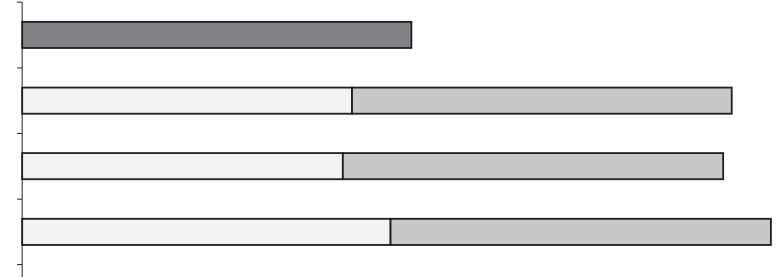

Con variable $\quad \square$ Sin variable $\quad \square$ Con todas las variables

Figura 4. Importancia relativa de las variables ambientales sobre la prueba de base Jackknife. Los valores muestran la contribución de cada variable. A. Regularized training gain; B. Test AUC.

Según los resultados obtenidos en el presente estudio, en la región de MM la probabilidad de la distribución de la enfermedad incrementa al aumentar la altitud, manteniéndose estable al superar los $639 \mathrm{msnm}$. En el caso de la precipitación, en el período más seco se evidencia que, la enfermedad se mantiene estable a precipitaciones de $27 \mathrm{~mm}$, mostrando un incremento al superar dicho valor y estabilizándose nuevamente de los $33 \mathrm{~mm}$ en adelante. La temperatura es otro de los factores determinantes en el desarrollo de la enfermedad en la zona, tanto para el trimestre más caluroso como el más frío. Las temperaturas promedio para los trimestres más calurosos y fríos indican que a menor temperatura existe mayor probabilidad de presencia de la enfermedad, la cual disminuye a partir de los 25 y $23^{\circ} \mathrm{C}$, respectivamente. La desviación estándar es baja para cada una de las variables ambientales, lo cual aumenta el nivel de confianza al evaluar la relación de las mismas con el desarrollo de la enfermedad (Fig. 5).

\section{Distribución potencial de la pudrición radicular del aguacate}

Este estudio evaluó el riesgo de la presencia de pudrición radicular en las poblaciones de árboles de aguacate en Montes de María, la principal región productora en el Caribe Colombiano. Esta estimación ha sido desarrollada con base en los datos de la presencia del síntoma de marchitamiento de yemas. Este síntoma mostró una alta correlación con la incidencia y la severidad de la enfermedad (Fig.1). El propósito general de las evaluaciones de enfermedades 

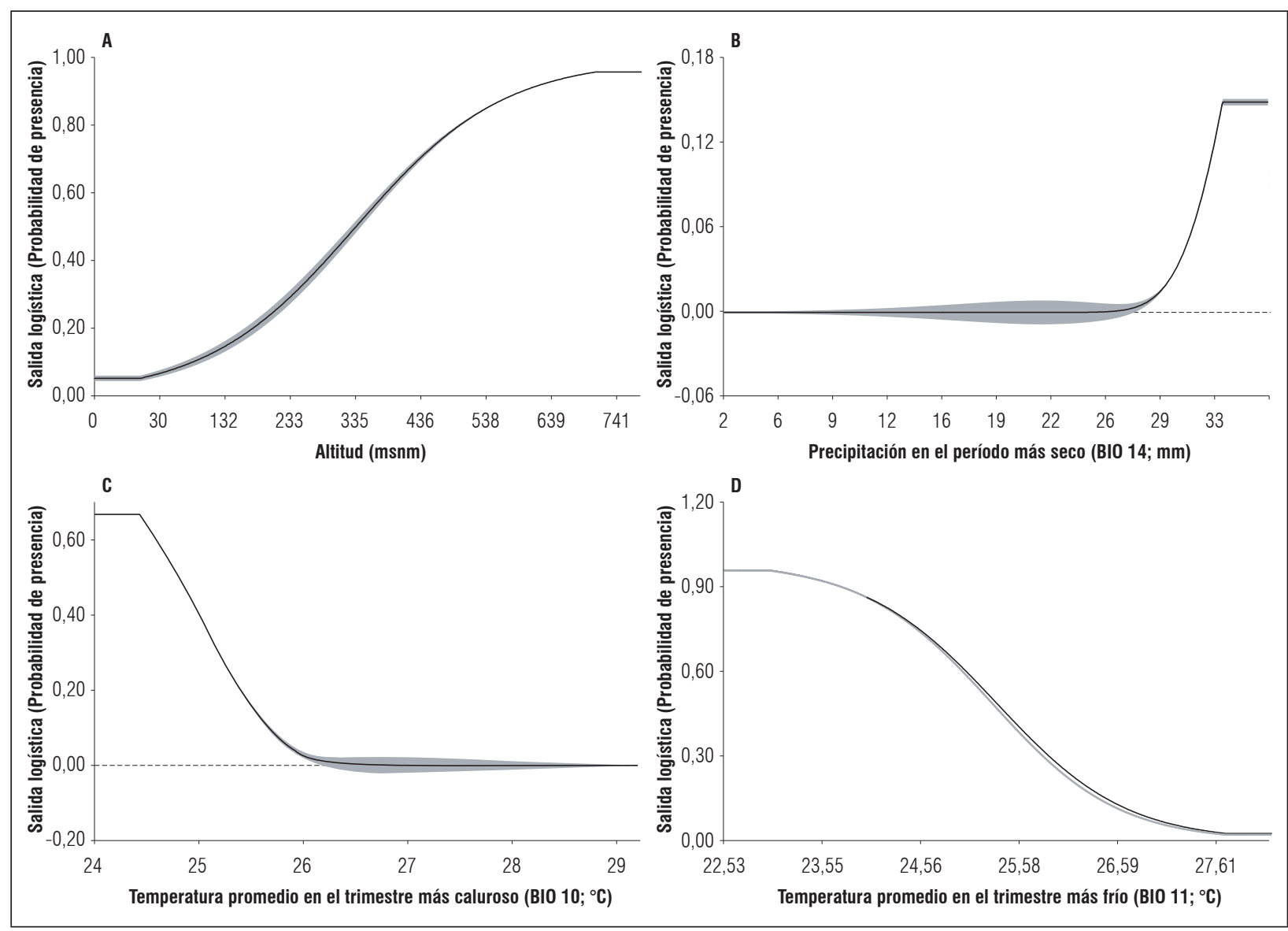

Figura 5. Curvas de respuesta de los mejores predictores para marchitamiento del aguacate en el modelo. A. Altitud; B. Precipitación en el período más seco (BIO 14; ${ }^{\circ} \mathrm{C}$ ); C. Temperatura promedio en el trimestre más caluroso (BIO 10; $\left.{ }^{\circ} \mathrm{C}\right) ; \mathrm{D}$. Temperatura promedio en el trimestre más frío (BIO 11; $\left.{ }^{\circ} \mathrm{C}\right)$. Las líneas negras son las curvas de respuesta promedio y los márgenes grises son las desviaciones estándar $( \pm)$, calculadas a partir de las 10 réplicas del modelo.

es proveer estimaciones confiables sobre el incremento de estas en un área y se fundamenta en la evaluación de síntomas y signos específicos característicos de un patógeno, al costo más bajo posible y con una confianza conocida. Lograr este propósito es clave en el desarrollo de esquemas de evaluación de enfermedades (Campbell y Neher, 1994). En el caso de la pudrición radicular, este estudio ha demostrado que la incidencia y la severidad de la pudrición radicular de árboles de aguacate en parcelas de agricultores pueden ser estimadas a partir de la frecuencia del síntoma de marchitamiento de yemas; el cual es un síntoma fácilmente distinguible y cuantificable, garantizando de esta manera un menor error de estimación en evaluaciones visuales. Adicionalmente este síntoma puede ser usado en la estandarización de protocolos basados en imágenes RGB.
El modelo Maxent obtenido, exhibe una alta validación estadística (test $A U C \geq 0,9$ ). Este modelo permite predecir las zonas (veredas) donde el riesgo de desarrollo de la pudrición radicular es más alto. Las variables que mayor efecto exhibieron sobre el modelamiento son altitud, precipitación en el período más seco (BIO14), temperatura promedio en el trimestre más caluroso (BIO10), temperatura promedio en el trimestre más frío (BIO11) y la temperatura media en el trimestre más lluvioso (BIO8). El efecto de la altitud y la precipitación sobre la distribución de la pudrición radicular es predecible en consideración a su efecto directo sobre la distribución de los árboles de aguacate lo cual delimita la probabilidad donde la enfermedad puede ser observada. El riesgo de desarrollo de la enfermedad incrementa proporcionalmente a la altitud entre los 200 a $400 \mathrm{~m}$ alcanzando el mayor riesgo en las mayores alturas $(\sim 741 \mathrm{~m})$. 
Las características de la región que exhibe el mayor riesgo de pudrición radicular son aquellas con la precipitación más alta durante la estación seca (BIO17 - BIO14), así como las temperaturas más bajas en los trimestres más fríos (BIO11 $\sim 22^{\circ} \mathrm{C}$ ) y calurosos (BIO10 $\sim 24^{\circ} \mathrm{C}$ ) (Fig. 5). El efecto de la precipitación y la temperatura han sido previamente identificados como variables asociadas a la presencia de pudrición radicular. El agente causal de la pudrición radicular es más efectivo en la reducción del crecimiento de la raíz a temperaturas de 21 a $27^{\circ} \mathrm{C}$, con un efecto importante en el borde inferior del rango descrito (Zentmyer, 1981). La temperatura de crecimiento micelial óptimo de este patógeno se encuentra entre los 24 a $27^{\circ} \mathrm{C}$, el cual se detiene por completo en temperaturas por debajo de los $10^{\circ} \mathrm{C}$ y por encima de los $33^{\circ} \mathrm{C}$ (Zentmyer et al., 1976; Shepherd y Pratt, 1974; Zentmyer, 1981).

Altas precipitaciones durante la estación seca provocan suelos húmedos durante la mayor parte del año. Este tipo de suelo es un factor muy importante en el incremento de la severidad y la diseminación de varias especies de Phytophthora (Erwin y Ribeiro, 1996). Modelos de predicción previos han sugerido un mayor riesgo de desarrollo de pudrición radicular con variables asociadas a suelos húmedos (La Manna et al., 2012). En el caso de P. cinnamomi, los esporangios solo son producidos en suelos húmedos, mientras la producción de clamidosporas no depende del nivel de humedad del suelo (Reeves, 1975). Adicionalmente, las zoosporas requieren que los poros del suelo están llenos de agua para poder nadar y facilitar la diseminación de la enfermedad (Aubrey, 1980)

Las variedades antillanas provienen de bosques secos tropicales de Centroamérica, donde predomina una estación lluviosa corta. Las condiciones climáticas encontradas en el presente estudio para la pudrición radicular son cercanas a las consideradas en Colombia como las ideales para el cultivo de variedades antillanas de aguacate (temperaturas con un rango de 18 a $26^{\circ} \mathrm{C}$ y alturas por debajo de los $1.000 \mathrm{msnm}$ ) (DANE, 2015). El resultado era el esperado en consideración a que los requerimientos climáticos del patógeno son muy similares a los del hospedero que parasita. Tal similitud ha sido reportada previamente en otros modelos de predicción de enfermedades (Galdino et al., 2016). El modelo obtenido para pudrición radicular muestra que una gran parte del área con aptitud para el establecimiento del cultivo brinda las condiciones óptimas para el desarrollo de la enfermedad de pudrición radicular, particularmente en el área central de Montes de María.
P. cinnamomi es un patógeno que puede permanecer durante largos periodos de tiempo en el suelo, impidiendo la erradicación efectiva en las áreas afectadas (Gallo-LLobet et al., 2006). En este escenario, las medidas de control cuarentenarias para evitar la dispersión de la pudrición radicular son innecesarias al considerar factores bióticos que claramente indican cuales son las áreas de mayor riesgo de diseminación del patógeno, así como la proximidad espacial de áreas de alto riesgo y menor riesgo. Sin embargo, es necesario que las áreas de baja prevalencia sean monitoreadas de manera rutinaria para determinar si la enfermedad ha cambiado su nivel de desarrollo. Adicionalmente, el reconocimiento de áreas con mayor potencial de desarrollo de la enfermedad puede ser usado por programas de selección de patrones tales como los desarrollados por Corpoica (Burbano-Figueroa, Comunicación personal). Los individuos sobrevivientes en áreas de mayor prevalencia de la enfermedad, posiblemente tienen mayor probabilidad de contar con genes de tolerancia o resistencia a la enfermedad y a condiciones de encharcamiento del suelo, característica utilizada en el desarrollo de patrones de la raza mexicana resistentes a pudrición radicular (Gabor, 1990; Pérez-Jiménez et al., 2008).

Los resultados de este estudio deben ser interpretados con precaución. En este estudio hemos usado Maxent por su facilidad de manejo, robustez en los modelos producidos y el relativo tamaño de la zona sobre la cual se desarrolló la predicción. No obstante, otras técnicas de modelamiento pueden ser usadas y en un futuro, una mayor cantidad de datos pueden ser incorporados para incrementar la precisión de las predicciones. Más allá de estas apreciaciones técnicas, modelos como estos son útiles en el diseño de estrategias racionales para evitar la introducción y dispersión de plagas y enfermedades, y desarrollar planes eficientes de manejo.

Es preciso tener en cuenta la importancia que han adquirido estos modelos geoespaciales predictivos en el área agrícola y aún más si son aplicados a localidades vulnerables a los efectos del cambio climático. Algunas zonas de los MM y su área de influencia, considerando predicciones climáticas disponibles, se encuentran expuestas a diversos efectos de la variabilidad climática. Estudios reportan que zonas con clima netamente de sabana tropical cambiaran a un clima ecuatorial monson (Am) y de un clima cálido y templado a uno completamente húmedo (Cf) (Rubel y Kottek, 2010). Según lo anterior, se evidencia una alteración de las condiciones climáticas del área de 
estudio, la cual podría tener influencia directa sobre el desarrollo de la enfermedad, esto, al ser favorecida por temperaturas inferiores a los $26^{\circ} \mathrm{C}$ y constantes precipitaciones.

En el caso de Montes de María, son crecientes los esfuerzos para atender las dificultades de los agricultores. Las parcelas fueron abandonadas por décadas y solo recuperadas en los últimos años cuando el conflicto colombiano ha llegado a su fin. El cultivo de aguacate representó uno de los principales ingresos para esas comunidades y hoy en día es considerado junto con el cacao, como las opciones más viables para el desarrollo de la región. La pudrición radicular es uno de los principales problemas en Montes de María; por consiguiente, la presente investigación como uno de los primeros estudios desarrollados en la estimación del riesgo de la enfermedad, contribuye a posibles planes de monitoreo, control y desarrollo de estrategias de manejo de P. cinnamomi en función de los escenarios de riesgo mostrados aquí.

\section{AGRADECIMIENTOS}

Esta investigación tuvo el apoyo financiero del Ministerio de Agricultura y Desarrollo Rural (Bogotá). La Corporación Colombiana de Investigación Agropecuaria (Corpoica) suministró el apoyo logístico necesario para su desarrollo.

Conflicto de intereses: el manuscrito fue preparado y revisado con la participación de los autores, quienes declaran no tener algún conflicto de interés que coloquen en riesgo la validez de los resultados aquí presentados.

\section{REFERENCIAS BIBLIOGRÁFICAS}

Aubrey, Z.G. 1980. Phytophthora cinnamomi and the diseases it causes. Amer. Phytopathol. Soc. Monogr. 10, 1-96.

Bogosian V., E. Hellgren, M. Sears y R. Moody. 2012. High-resolution niche models via a correlative approach: Comparing and combining correlative and process-based information. Ecol. Model. 337-238, 63-73. Doi: 10.1016/j.ecolmodel.2012.04.017

Burns, R.M., J.H. Miner, C.D. Gustafson, G.A. Zentmyer y W.A. Thorn. 1960. Correlation of soil series and avocado root rot damage in the Fallbrook area. Calif. Avoc. Soc. 44, 110-13.

Campbell, C.L. y D.A. Neher. 1994. Estimating disease severity and incidence. pp. 117-47. En: Campbell, L. y M. Benson (eds.). Epidemiology and management of root diseases. Springer Verlag, Berlin, Heidelberg, Alemania. Doi: 10.1007/978-3-642-85063-9_5

Castillo R., C.J. 2008. Modelamiento de la distribución de los nichos adecuados para la invasión biológica del retamo espinoso (Ilex europaeus) en la cuenca alta del Río Bogotá, vulnerabilidad y escenarios futuros. Tesis de doctorado. Pontificia Universidad Javeriana, Bogotá, Colombia.

Chen, H., P.L. Morrell, V. Ashworth, M. de la Cruz y M.T. Clegg. 2009. Tracing the geographic origins of major avocado cultivars. J. Hered. 100(1), 56-65. Doi: 10.1093/jhered/esn068

Chinchilla, M., R. Mata y A. Alvarado. 2011. Andisoles, Inceptisoles y Entisoles de la subcuenca del río Pirrís, región de los Santos, Talamanca, Costa Rica. Agron. Costarricense 35(1), 83-107.

Coffey, M.D. 1987. Phytophthora root rot of avocado: an integrated approach to control in California. Plant Dis. 71(11), 1046-1052

DANE (Departamento Administrativo Nacional de Estadística). 2015. El cultivo del aguacate (Persea americana Miller), fruta de extraordinarias propiedades alimenticias, curativas e industriales (Primera parte). Boletín Mensual Insumos y Factores Asociados a la Producción Agropecuaria 40. Bogotá, Colombia.

Darvas, J.M., J.C. Toerien y D.L. Milne. 1983. Injection of established avocado trees for the effective control of Phytophthora root rot. SAAGA 6, 76-77.

Darvas J.M., J.C. Toerien y D.L. Milne. 1984. Control of avocado root rot by trunk injection with phosethyl-A1. Plant Dis. 68, 691-693.

Erwin D.C. y O.K. Ribeiro. 1996. Phytophthora diseases worldwide. American Phytopathological Society Press, St. Paul, MN, USA.

Gabor, B.K. 1990. Quantitative analysis of the resistance to Phytophthora cinnamomi in five avocado rootstocks under greenhouse conditions. Plant Dis. 74(11), 882-885. Doi: 10.1094/PD-74-0882

Galdino T.V. da S., S. Kumar, L.S.S. Oliveira, A.C. Alfenas, L.G. Neven, A.M. Al-Sadi y M. Picanço .2016. Mapping global potential risk of mango sudden decline disease caused by Ceratocystis fimbriata. PLoS ONE 11(7). Doi: 10.1371/journal.pone.0159450

Gallo-Llobet, L., A. Baños-Atance y A. Rodríguez-Pérez. 2006. Selección de patrones de aguacate de raza antillana tolerante-resistentes a Phytophthora cinnamomi para el control de la podredumbre de raíz. p. 238. Libro de Resúmenes, XIII Congreso de la Sociedad Española de Fitopatología, Murcia, España.

Guevara V.M., M. Vertel M., A. Castellar M. y P. Blanco T. 2016. Distribución geográfica de especies de garrapatas infectadas con Babesia de zonas rurales del 
departamento de Sucre, Colombia. XXVI Simposio Internacional de Estadística, Sincelejo, Colombia.

Hijmans, R.J., S. Cameron y J. Parra. 2016. WorldClim. En: http://www.worldclim.org/version1; consulta: febrero de 2017.

Hijmans, R.J., S.E. Cameron, J.L. Parra, P.G. Jones y A. Jarvis. 2005. Very high resolution interpolated climate surfaces for global land areas. Int. J. Climatol. 25(15), 1965-78. Doi: 10.1002/joc.1276

Jiménez-Valverde, A., J.M. Loboy J. Hortal. 2008. Not as good as they seem: the importance of concepts in species distribution modelling. Diversity and Distributions 14(6), 885-90. Doi: 10.1111/j.1472-4642.2008.00496.x

Jiménez-Valverde, A., A.T. Peterson, J. Soberón, J.M. Overton, P. Aragón y J.M. Lobo. 2011. Use of niche models in invasive species risk assessments. Biol. Invasions 13(12), 2785-97. Doi: 10.1007/s10530-011-9963-4

Kottek, M., J. Grieser, C. Beck, B. Rudolph y F. Rubel. 2006. World Map of the Köppen-Geiger climate classification updated. Meteor. Zeitschr. 15(3), 259-63. Doi: 10.1127/0941-2948/2006/0130

Kumar, S., L.G. Neven y W.L. Yee. 2014. Evaluating correlative and mechanistic niche models for assessing the risk of pest establishment. Ecosphere 5(7), 1-23. Doi: 10.1890/ES14-00050.1

La Manna, L., S.D. Matteucci y T. Kitzberger. 2012. Modelling Phytophthora disease risk in Austrocedrus chilensis forests of Patagonia. Eur. J. For. Res. 131(2), 323-337. Doi: 10.1007/s10342-011-0503-7

Manuel, S., H.C. Williams y S.J. Ormerod. 2002. Evaluating presence-absence models in ecology: the need to account for prevalence. J. Appl. Ecol. 38(5), 921-931. Doi: 10.1046/j.1365-2664.2001.00647.x

Pavas, T., C.A. 2015. Organización de cadena productiva del aguacate. Consejo Nacional del Aguacate, Ministerio de Agricultura, Bogotá, Colombia.

Pegg, K.G., L.M. Coates, L. Korsten y R.M. Hardig. 2002. Foliar, fruit and soilborne diseases. $2^{\text {nd }}$ ed. pp. 299339. En: Schaffer, B., B.N. Wolstenholme y A.W. Whiley (eds.). The avocado: Botany, production and uses. Wallingford, Oxon, UK. Doi: 10.1079/9780851993577.0299
Pérez-Jiménez, R. 2008. Significant avocado diseases caused by fungi and oomycetes. Eur. J. Plant Sci. Biotechnol. $2(1), 1-24$.

Phillip, S.J., R.P. Anderson y R.E. Schapire. 2005. Maximum entropy modeling of species geographic distributions. Ecol. Model. 190, 231-259.

Reeves, J.R. 1975. Behaviour of Phytophthora cinnamomi Rands in different soils and water regimes. Soil Biol. Biochem. 7(1), 19-24. Doi: 10.1016/0038-0717(75)90025-5

Rubel, F. y M. Kottek. 2010. Observed and projected climate shifts 1901-2100 depicted by world maps of the Koppen-Geiger climate classification. Meteorol. Zeitschr. 19(2), 135-41. Doi: 10.1127/0941-2948/2010/0430

Shepherd, C.J. y B.H. Pratt. 1974. Temperature-growth relations and genetic diversity of A2 mating-type isolates of Phytophthora cinnamomi in Australia. Aust. J. Bot. 22(2), 231-49. Doi: 10.1071/BT9740231

Tamayo, A., O. Córdoba y M.E. Londoño. 2008. Tecnología para el cultivo del aguacate. Vol. 5. Corpoica, Río Negro, Colombia.

Téllez, A. 2015. Sistematización de la experiencia de Colombia responde en la zona de consolidación territorial de los Montes de María. Funicar, Cartagena, Colombia.

Vega, J. 2012. El aguacate en Colombia: Estudio de caso de los Montes de María, en el Caribe colombiano. Banco de República - Economía Regional 171, 1-145.

Zentmyer, G.A. 1981. The effect of temperature on growth and pathogenesis of Phytophthora cinnamomi and on growth of its avocado host. Phytopathology 71, 92528. Doi: 10.1094/Phyto-71-925.

Zentmyer, G.A. 1984. Avocado diseases. Int. J. Pest Manag. 30(4), 388-400. Doi: 10.1080/09670878409370915

Zentmyer, G.A., J.V. Leary, L.J. Klure y G.L. Grantham. 1976. Variability in growth of Phytophthora cinnamomi in relation to temperature. Phytopathology 66, 982986. Doi: 10.1094/Phyto-66-982

Zentmyer, G.A. y S.M. Mircetich. 1967. Saprophytism and persistence in soil by Phytophthora cinnamomi. Phytopathology 51, 117-24. 